\title{
BOUNDEDNESS PROPERTIES OF RESOLVENTS AND SEMIGROUPS OF OPERATORS
}

\author{
J. A. VAN CASTEREN \\ University of Antwerp, Department of Mathematics and Computer Science \\ Universiteitsplein 1, 2610 Antwerp/Wilrijk, Belgium \\ E-mail: vcaster@uia.ua.ac.be
}

\begin{abstract}
Let $T: \mathcal{H} \rightarrow \mathcal{H}$ be an operator in the complex Hilbert space $\mathcal{H}$. Suppose that $T$ is square bounded in average in the sense that there exists a constant $M(T)$ with the property that, for all natural numbers $n$ and for all $x \in \mathcal{H}$, the inequality

$$
\frac{1}{n+1} \sum_{j=0}^{n}\left\|T^{j} x\right\|^{2} \leq M(T)^{2}\|x\|^{2}
$$

is satisfied. Also suppose that the adjoint $T^{*}$ of the operator $T$ is square bounded in average with constant $M\left(T^{*}\right)$. Then the operator $T$ is power bounded in the sense that $\sup \left\{\left\|T^{n}\right\|: n \in \mathbb{N}\right\}$ is finite. In fact the following inequality is valid for all $n \in \mathbb{N}$ :
\end{abstract}

$$
\left\|T^{n}\right\| \leq e M(T) M\left(T^{*}\right) .
$$

Suppose that $T$ has a bounded everywhere defined inverse $S$ with the property that for $\lambda$ in the open unit disc of $\mathbb{C}$ the operator $(I-\lambda S)^{-1}$ exists and that the expression

$$
\sup \left\{(1-|\lambda|)\left\|(I-\lambda S)^{-1}\right\|:|\lambda|<1\right\}
$$

is finite. If $T$ is power bounded, then so is $S$ and hence in such a situation the operator $T$ is similar to a unitary operator. If both the operators $T^{*}$ and $S$ are square bounded in average, then again the operator $T$ is similar to a unitary operator. Similar results hold for strongly

1991 Mathematics Subject Classification: 47A30, 47D05.

Key words and phrases: power bounded operator, bounded semigroup, operator Poisson kernel, square bounded in average.

The author is grateful to a number of people who in one way or another were involved during the preparation of this paper: F. Delbaen, L. Waelbroeck (Brussels), J. Zemánek (Warsaw). The author also wants to thank the National Fund for Scientific Research (NFWO) and the University of Antwerp (UIA) for their material support. The author is indebted to the referee for pointing out some errors in an earlier draft of the paper. Finally, the author is obliged to the "International Scientific and Technical Cooperation BLEU-Poland" for making it possible to visit the Banach Center in Warsaw in April 1994.

The paper is in final form and no version of it will be published elsewhere. 
continuous semigroups instead of (powers) of a single operator. Some results are also given in the more general Banach space context.

1. Introduction. In this paper we want to discuss the claims stated in the abstract. To some extent we continue the discussions of $[26,27,28]$, where, among others, results closely related to the ones in the abstract are proved. To the best knowledge of the author Propositions 2.1, 2.2,3.1, and 3.2 are new and consequently the presentation of the proofs of Theorems 2.3 and 3.3 is new as well. We will need some rather elementary inequalities: for a proof see e.g. [27, Lemma 1.1].

1.1. Lemma. Let $\mu$ be a non-negative Borel measure on the positive half axis. The following inequalities are valid:

$$
\begin{aligned}
& \frac{1}{e} \sup _{t>0} \frac{\mu[0, t]}{t+1} \leq \sup _{0<r<1}(1-r) \int_{0}^{\infty} r^{s} d \mu(s) \leq \sup _{t>0} \frac{\mu[0, t)}{t} \quad \text { and } \\
& \frac{1}{e} \sup _{t>0} \frac{\mu[0, t]}{t} \leq \sup _{\omega>0} \omega \int_{0}^{\infty} e^{-\omega s} d \mu(s) \leq \sup _{t>0} \frac{\mu[0, t)}{t} .
\end{aligned}
$$

1.2. LEMMA. Let $h$ be a complex valued harmonic function on the right complex half plane for which

$$
M_{0}:=\sup \left\{\int_{-\infty}^{\infty}|h(\omega, \xi)| d \xi: \omega>0\right\}
$$

is finite. The following assertions hold true:

(a) The function $h$ satisfies the following inequality:

$$
3 \pi \omega|h(\omega, \xi)| \leq 4 M_{0}, \quad \omega>0, \xi \in \mathbb{R}
$$

(b) There exists a complex measure $\mu$ on $\mathbb{R}$, which is of bounded variation, such that

$$
h(\omega, \xi)=\frac{\omega}{\pi} \int_{-\infty}^{\infty} \frac{1}{\omega^{2}+(\xi-\eta)^{2}} d \mu(\eta), \quad \omega>0, \xi \in \mathbb{R} .
$$

(c) Suppose that $h$ is of the form

$$
h(\omega, \xi)=F(\omega+i \xi)+G(\omega-i \xi), \quad \omega>0, \xi \in \mathbb{R},
$$

where the functions $F$ and $G$ are holomorphic and where

$$
\sup \{\omega|F(\omega)|: \omega>0\}
$$

is finite. Then

$$
\begin{array}{ll}
F(\lambda)=\frac{1}{2 \pi} \int_{-\infty}^{\infty} \frac{d \mu(\lambda)}{\lambda-i \eta}, \quad \Re \lambda>0, \quad \text { and } \\
G(\lambda)=\frac{1}{2 \pi} \int_{-\infty}^{\infty} \frac{d \mu(\lambda)}{\lambda+i \eta}, \quad \Re \lambda>0 .
\end{array}
$$

Pro of. (a) The reader is referred to Duren [6, Lemma 1, p. 188].

(b) The reader may consult Stein [21, Theorem 2, Corollary, p. 200].

(c) The reader is referred to [27, Lemma 1.2.]. 
2. Power bounded operators and operators similar to unitary ones. In this section the operators $T$ and $S$ are everywhere defined continuous linear operators in the Hilbert space $\mathcal{H}$. In (2.1) and (2.3) below we assume that the operator $T$ has its spectrum in the closed unit circle of $\mathbb{C}$ and in (2.2) it is assumed that the spectrum of $T$ is a subset of the unit circle in $\mathbb{C}$ and that $S T=T S=I$. Moreover $0<|\lambda|<1$ in $(2.2)$ and $|\lambda|<1$ in (2.3). The following equalities do part of the work (the curve $\gamma_{r}$ is the positively oriented circle of radius $r, 0<r<1)$ :

$$
\begin{gathered}
(n+1)\left\langle T^{n} x, y\right\rangle=\frac{1}{2 \pi i} \int_{\gamma_{r}}\left\langle(I-\lambda T)^{-2} x, y\right\rangle \frac{d \lambda}{\lambda^{n+1}} \\
(I-\lambda S)^{-1}=\frac{1}{\lambda}\left\{\left(1-|\lambda|^{2}\right)(I-\lambda S)^{-1}-I\right\} T(I-\bar{\lambda} T)^{-1} \\
(I-\lambda T)^{-1}=\frac{1}{2 \pi i} \int_{-\pi}^{\pi} e^{i \vartheta}\left(I+e^{i \vartheta} \lambda T\right)^{-2} \vartheta d \vartheta .
\end{gathered}
$$

The equality in $(2.1)$ is true because $(I-\lambda T)^{-2}=\sum_{k=1}^{\infty} k(\lambda T)^{k-1}$, and the equality in $(2.3)$ is correct for the same reason together with integration by parts.

Definition. The operator $T$ is said to be square bounded in average with a finite constant $M(T)$ if the inequality

$$
\sum_{j=0}^{n}\left\|T^{j} x\right\|^{2} \leq M(T)^{2}(n+1)\|x\|^{2}
$$

is valid for all $n \in \mathbb{N}$ and for all $x \in \mathcal{H}$.

In the following proposition we will see that an operator $T$ which itself is square bounded in average and for which $T^{*}$ is square bounded in average as well, is necessarily power bounded in the sense that $\sup \left\{T^{n}: n \in \mathbb{N}\right\}<\infty$.

2.1. Proposition. Suppose that both $T$ as well as $T^{*}$ are square bounded in average with constants $M(T)$ and $M\left(T^{*}\right)$ respectively. Then $T$ is power bounded and

$$
\sup _{n \in \mathbb{N}}\left\|T^{n}\right\| \leq e M(T) M\left(T^{*}\right) .
$$

Proof. Fix $x, y \in \mathcal{H}$, fix $0<r<1$ and consider (see (2.1))

$$
\begin{aligned}
(n+1) r^{n}\left(1-r^{2}\right)\left\langle T^{n} x, y\right\rangle & =\frac{1-r^{2}}{2 \pi} \int_{-\pi}^{\pi} e^{-i n \vartheta}\left\langle\left(I-r e^{i \vartheta} T\right)^{-2} x, y\right\rangle d \vartheta \\
& =\frac{1-r^{2}}{2 \pi} \int_{-\pi}^{\pi} e^{-i n \vartheta}\left\langle\left(I-r e^{i \vartheta} T\right)^{-1} x,\left(I-r e^{-i \vartheta} T^{*}\right)^{-1} y\right\rangle d \vartheta .
\end{aligned}
$$

Then

$$
(n+1) r^{n}\left(1-r^{2}\right)\left|\left\langle T^{n} x, y\right\rangle\right| \leq \frac{1-r^{2}}{2 \pi} \int_{-\pi}^{\pi}\left|\left\langle\left(I-r e^{i \vartheta} T\right)^{-1} x,\left(I-r e^{-i \vartheta} T\right)^{-1} y\right\rangle\right| d \vartheta
$$




$$
\begin{aligned}
\leq & \frac{1-r^{2}}{2 \pi} \int_{-\pi}^{\pi}\left\|\left(I-r e^{i \vartheta} T\right)^{-1} x\right\| \cdot\left\|\left(I-r e^{-i \vartheta} T^{*}\right)^{-1} y\right\| d \vartheta \\
\leq & \left(1-r^{2}\right)\left(\frac{1}{2 \pi} \int_{-\pi}^{\pi}\left\|\left(I-r e^{i \vartheta} T\right)^{-1} x\right\|^{2} d \vartheta\right)^{1 / 2} \\
& \times\left(\frac{1}{2 \pi} \int_{-\pi}^{\pi}\left\|\left(I-r e^{-i \vartheta} T^{*}\right)^{-1} y\right\|^{2} d \vartheta\right)^{1 / 2} \\
= & \left(1-r^{2}\right)\left(\sum_{n=0}^{\infty} r^{2 n}\left\|T^{n} x\right\|^{2}\right)^{1 / 2}\left(\sum_{n=0}^{\infty} r^{2 n}\left\|\left(T^{*}\right)^{n} y\right\|^{2}\right)^{1 / 2} \\
\leq & \left(\sup _{n \in \mathbb{N}} \frac{1}{n+1} \sum_{j=0}^{n}\left\|T^{j} x\right\|^{2}\right)^{1 / 2}\left(\sup _{n \in \mathbb{N}} \frac{1}{n+1} \sum_{j=0}^{n}\left\|\left(T^{*}\right)^{j} y\right\|^{2}\right)^{1 / 2} .
\end{aligned}
$$

Choose $r^{2}=n /(n+2)$ and the result in Proposition 2.1 will follow.

R e m a r k. If we set $r^{2}=n /(n+1)$ in the proof of Proposition 2.1 we get the inequality

$$
\left\|T^{n}\right\| \leq\left(\frac{n+1}{n}\right)^{n} \frac{n+1}{2 n+1} M(T) M\left(T^{*}\right) \leq e \frac{n+1}{2 n+1} M(T) M\left(T^{*}\right) .
$$

In the remainder of this section $S$ and $T$ are supposed to be everywhere defined operators in a Hilbert space $\mathcal{H}$ with the property that $S T=T S=I$. It is also assumed that the spectrum of $T$ and hence also that of $S$ is a subset of the unit circle $\{\lambda \in \mathbb{C}$ : $|\lambda|=1\}$.

2.2. Proposition. Suppose that $M_{1}$ defined by

$$
M_{1}:=\sup \left\{\frac{1}{|\lambda|}\left\|\left[\left(1-|\lambda|^{2}\right)(I-\lambda S)^{-1}-I\right] T\right\|: 0<|\lambda|<1\right\}
$$

is finite. Suppose also that $S T=T S=I$. For $0 \leq r<1$ and $x \in \mathcal{H}$, the following inequalities are true:

$$
\begin{gathered}
\sum_{n=0}^{\infty} r^{2 n}\left\|S^{n} x\right\|^{2} \leq M_{1}^{2} \sum_{n=0}^{\infty} r^{2 n}\left\|T^{n} x\right\|^{2} \quad \text { and } \\
\sum_{n=0}^{\infty} r^{2 n}\left\|\left(S^{*}\right)^{n} x\right\|^{2} \leq M_{1}^{2} \sum_{n=0}^{\infty} r^{2 n}\left\|\left(T^{*}\right)^{n} x\right\|^{2} .
\end{gathered}
$$

Proof. For $|\lambda|<1$ and $x \in \mathcal{H}$, the following inequality is valid (see (2.2)):

$$
\left\|(I-\lambda S)^{-1} x\right\| \leq M_{1}\left\|(I-\bar{\lambda} T)^{-1} x\right\| .
$$

It follows that

$$
\begin{aligned}
\sum_{n=0}^{\infty} r^{2 n}\left\|S^{n} x\right\|^{2} & =\frac{1}{2 \pi} \int_{-\pi}^{\pi}\left\|\left(I-r e^{i \vartheta} S\right)^{-1} x\right\|^{2} d \vartheta \\
& \leq M_{1}^{2} \frac{1}{2 \pi} \int_{-\pi}^{\pi}\left\|\left(I-r e^{-i \vartheta} T\right)^{-1} x\right\|^{2} d \vartheta=M_{1}^{2} \sum_{n=0}^{\infty} r^{2 n}\left\|T^{n} x\right\|^{2} .
\end{aligned}
$$


By the same token we have

$$
\sum_{n=0}^{\infty} r^{2 n}\left\|\left(S^{*}\right)^{n} x\right\|^{2} \leq M_{1}^{2} \sum_{n=0}^{\infty} r^{2 n}\left\|\left(T^{*}\right)^{n} x\right\|^{2} .
$$

The following theorem is taken from [1]. Two operators $T_{1}$ and $T_{2}$ are said to be similar if there exists a bounded linear operator $V$ with a bounded linear inverse, that is everywhere defined, such that $V T_{1}=T_{2} V$.

2.3. ThEOREM. The following assertions are equivalent:

(i) The operator $T$ is similar to a unitary operator;

(ii) The operators $T$ and $S$ are power bounded;

(iii) The operator $T$ is power bounded, the inverses $(I-\lambda S)^{-1},|\lambda|<1$, exist and

$$
\sup \left\{(1-|\lambda|)\left\|(I-\lambda S)^{-1}\right\|:|\lambda|<1\right\} \quad \text { is finite; }
$$

(iv) The operators $T$ and $T^{*}$ are square bounded in average and the expression in (2.5) is finite;

(v) The operators $S$ and $T^{*}$ are square bounded in average;

(vi) For every $x$ and $y \in \mathcal{H}$ the expression

$$
\sup _{0<r<1}\left(1-r^{2}\right) \int_{-\pi}^{\pi}\left|\left\langle\left(I-r e^{-i \vartheta} T\right)^{-1}\left(I-r e^{i \vartheta} S\right)^{-1} x, y\right\rangle\right| d \vartheta
$$

is finite.

Remark 1. The proof of the equivalence of (ii) and (iii) is contained in $[26$, Theorem 1]. In [26] more references can be found as well. The present result is closely related to a problem posed by Sz.-Nagy in [9, p. 585]. Another source of information is Sz.Nagy and Foiaş [25, p. 334], where the relation with characteristic operator functions is explained and where a weaker form of the present theorem is proved in the context of dilation theory of contraction operators. Another closely related paper is Stampfli [20]. For a contraction operator the equivalence of (ii) and (iii) is due to Gohberg and Krein [10], who deduced it from a theorem of Sz.-Nagy and Foiaş [24]. In the latter paper the authors provide a sufficient condition for an invertible contraction operator $T$ to be similar to a unitary operator in terms of the characteristic operator function $\Theta_{T}(\lambda):=\left.\left[-T+\lambda D_{T^{*}}\left(I-\lambda T^{*}\right)^{-1} D_{T}\right]\right|_{\mathcal{D}_{T}}$ of $T$. This condition requires the existence of a constant $N$ for which

$$
\|x\| \leq N\left\|\Theta_{T}(\lambda) x\right\|, \quad|\lambda|<1, x \in \mathcal{D}_{T} .
$$

Here $D_{T}=\sqrt{I-T^{*} T}$ and $\mathcal{D}_{T}$ is the closure of the range of $D_{T}$.

Remark 2. In [32] and [17] Mbekhta and Zemánek are interested in the limit behavior of the averages $M_{n}(T)=(n+1)^{-1} \sum_{k=0}^{n} T^{k}$. It is not so clear how their results compare to ours. Zemánek is interested in conditions that force an operator to be the identity: see [32]. In fact in view of Theorem 1.12(c), p. 523, in Bennett et al. [3] and Remark 1 below, a direct relation does not seem to exist.

Proof of Theorem 2.3. The proof of the equivalence (i) and (ii) appears in Sz.-Nagy [23]. The implication (ii) $\Rightarrow$ (iii) is easy and the implication (iii) $\Rightarrow$ (iv) is trivial. 
The implication (iv) $\Rightarrow(\mathrm{v})$ is an immediate consequence of Proposition 2.2. A proof of $(\mathrm{v}) \Rightarrow(\mathrm{vi})$ may be given along the same lines as that of Proposition 2.1. For the proof the implication $(\mathrm{vi}) \Rightarrow$ (ii) we notice for $n \in \mathbb{Z}$ and for $x$ and $y$ in $\mathcal{H}$ the following identity:

$$
r^{|n|}\left\langle T^{n} x, y\right\rangle=\frac{1-r^{2}}{2 \pi} \int_{-\pi}^{\pi} e^{i n \vartheta}\left\langle\left(I-r e^{-i \vartheta} T\right)^{-1}\left(I-r e^{i \vartheta} S\right)^{-1} x, y\right\rangle d \vartheta .
$$

With $r=|n|(|n|+1)^{-1}$ we infer

$$
\left|\left\langle T^{n} x, y\right\rangle\right| \leq e \sup _{0<r<1} \frac{1-r^{2}}{2 \pi} \int_{-\pi}^{\pi}\left|\left\langle\left(I-r e^{-i \vartheta} T\right)^{-1}\left(I-r e^{i \vartheta} S\right)^{-1} x, y\right\rangle\right| d \vartheta .
$$

So $\sup \left\{\left|<T^{n} x, y\right\rangle \mid: n \in \mathbb{Z}\right\}$ is finite for every $x$ and $y$ in $\mathcal{H}$. By a Banach-Steinhaus argument it then follows that $\sup \left\{\left\|T^{n}\right\|: n \in \mathbb{Z}\right\}$ is finite.

ExAmple. Fix $0<2 \gamma<1$ and put $\alpha_{k}=(1+|k|)^{-\gamma}, k \in \mathbb{Z}$. Define the operator $T: \ell^{2}(\mathbb{Z}) \rightarrow \ell^{2}(\mathbb{Z})$ by $T e_{k}=\left(\alpha_{k+1} / \alpha_{k}\right) e_{k+1}, k \in \mathbb{Z}$. Then

$$
\sup _{n \in \mathbb{N}} \frac{1}{2 n+1} \sum_{j=-n}^{n}\left\|T^{j} x\right\|^{2} \leq\|x\|^{2} \sup _{k \in \mathbb{Z}, n \in \mathbb{N}} \frac{1}{(2 n+1) \alpha_{k}^{2}} \sum_{j=-n}^{n} \alpha_{k+j}^{2}<\infty .
$$

However, since $\left\|T^{j}\right\|=(1+|j|)^{\gamma}$, the operator $T$ cannot be similar to a unitary one.

3. Bounded semigroups and operators similar to self-adjoint ones. In this section the symbol $A$ stands for a closed linear operator with domain and range in a Hilbert space $\mathcal{H}$. The operator $i A$ is said to generate a strongly continuous semigroup $\{P(t): t \geq 0\}$ if

$$
i A=\mathrm{s}-\lim _{t \downarrow 0} \frac{P(t)-I}{t} .
$$

Occasionally the operator $P(t)$ is written as $P(t)=\exp (i t A)$. The operator $i A$ is said to generate a strongly continuous group if

$$
i A=\mathrm{s}-\lim _{t \rightarrow 0} \frac{P(t)-I}{t} .
$$

Again sometimes we write $P(-t)=\exp (-i t A)$. For more information on strongly continuous semigroups see e.g. Yosida [31, Chapter IX]. For the proof of Proposition 3.1 and Theorem 3.3 below we need Stone's representation theorem; see Yosida [31, Corollary 2, p. 253]. Furthermore we shall use Plancherel's Theorem in $L^{2}(R, \mathcal{H})$ : see Edwards and Gaudry [7, §3.4, p. 53] or Stein [21, Chapter 1, pp. 45-47]. The following identities are relevant. In (3.1) and in (3.3) it is assumed that the spectrum of the operator $i A$ is contained in the closed half plane $\{\lambda \in \mathbb{C}: \Re \lambda \leq 0\}$ and in (3.2) we assume that the spectrum is a subset of the purely imaginary axis $\{\lambda \in \mathbb{C}: \Re \lambda=0\}$. We write $\Gamma_{\omega}$ for the straight line $\Gamma_{\omega}(\xi)=\omega+i \xi$. Under these conventions we have:

$$
\begin{aligned}
t P(t) & =\frac{1}{2 \pi i} \int_{\Gamma_{\omega}} e^{z t}(z I-i A)^{-2} d z \\
(\lambda I-i A)^{-1} & =\left[2 \Re \lambda(\lambda I-i A)^{-1}-I\right](\bar{\lambda} I+i A)^{-1} \\
(\lambda I-i A)^{-1} & =i \int_{0}^{\infty}((\lambda+i \xi) I-i A)^{-2} d \xi .
\end{aligned}
$$


Definition. The semigroup $\{P(t)=\exp (i t A): t \geq 0\}$ is said to be average square bounded or square bounded in average with constant $M(i A)$ if the inequality

$$
\int_{0}^{t}\|P(s) x\|^{2} d s \leq t M(i A)^{2}\|x\|^{2}
$$

is valid for all $t>0$ and for all $x \in \mathcal{H}$.

The following proposition is the semigroup analogue to Proposition 2.1.

3.1. Proposition. Suppose that the semigroups

$$
\{P(t)=\exp (i t A): t \geq 0\} \quad \text { and } \quad\left\{P(t)^{*}=\exp \left(-i t A^{*}\right): t \geq 0\right\}
$$

are square bounded in average with constants $M(i A)$ and $M\left(-i A^{*}\right)$ respectively. Then the semigroup $\{P(t): t \geq 0\}$ is bounded in the sense that

$$
\|P(t)\| \leq \frac{e}{2} M(i A) M\left(-i A^{*}\right), \quad t>0 .
$$

Proof. Fix $t$ and $\omega>0$ and fix $x$ and $y$ in $\mathcal{H}$. From (3.1) we then infer

$$
\begin{aligned}
t\langle P(t) x, y\rangle & =\frac{e^{\omega t}}{2 \pi} \int_{-\infty}^{\infty} e^{i \xi t}\left\langle((\omega+i \xi) I-i A)^{-2} x, y\right\rangle d \xi \\
& =\frac{e^{\omega t}}{2 \pi} \int_{-\infty}^{\infty} e^{i \xi t}\left\langle((\omega+i \xi) I-i A)^{-1} x,\left((\omega-i \xi) I+i A^{*}\right)^{-1} y\right\rangle d \xi
\end{aligned}
$$

and so, using the Cauchy-Schwarz inequality,

$$
\begin{aligned}
t|\langle P(t) x, y\rangle| \leq & \frac{e^{\omega t}}{2 \pi} \int_{-\infty}^{\infty}\left|\left\langle((\omega+i \xi) I-i A)^{-1} x,\left((\omega-i \xi) I+i A^{*}\right)^{-1} y\right\rangle\right| d \xi \\
\leq & \frac{e^{\omega t}}{2 \pi} \int_{-\infty}^{\infty}\left\|((\omega+i \xi) I-i A)^{-1} x\right\|\left\|\left((\omega-i \xi) I+i A^{*}\right)^{-1} y\right\| d \xi \\
\leq & \frac{e^{\omega t}}{2 \pi}\left(\int_{-\infty}^{\infty}\left\|((\omega+i \xi)-i A)^{-1} x\right\|^{2} d \xi\right)^{1 / 2}\left(\int_{-\infty}^{\infty}\left\|\left((\omega-i \xi)+i A^{*}\right)^{-1} y\right\|^{2} d \xi\right)^{1 / 2} \\
= & e^{\omega t}\left(\int_{0}^{\infty} e^{-2 \omega s}\|P(s) x\|^{2} d s\right)^{1 / 2}\left(\int_{0}^{\infty} e^{-2 \omega s}\left\|P(s)^{*} y\right\|^{2} d s\right)^{1 / 2} \\
= & \frac{e^{\omega t}}{2 \omega}\left((2 \omega)^{2} \int_{0}^{\infty} e^{-2 \omega s} \int_{0}^{s}\|P(\sigma) x\|^{2} d \sigma d s\right)^{1 / 2} \\
& \times\left((2 \omega)^{2} \int_{0}^{\infty} e^{-2 \omega s} \int_{0}^{s}\left\|P(\sigma)^{*} y\right\|^{2} d \sigma d s\right)^{1 / 2} \\
\leq & \frac{e^{\omega t}}{2 \omega} M(i A) M\left(-i A^{*}\right) .
\end{aligned}
$$

(Plancherel's Theorem in Hilbert space)

The choice $\omega=t^{-1}$ yields the inequality in (3.5).

In what follows we assume that the spectrum of the operator $i A$ is a subset of the imaginary axis. As the semigroup analogue to Proposition 2.2 we have the following result. 
3.2. Proposition. Suppose that the quantity $M_{2}$ defined by

$$
M_{2}=\sup \left\{\left\|2 \Re \lambda(\lambda I-i A)^{-1}-I\right\|: \Re \lambda>0\right\}
$$

is finite. Then the following inequalities are valid:

$$
\begin{aligned}
& \int_{0}^{\infty} e^{-2 \omega s}\|P(s) x\|^{2} d s \leq M_{2}^{2} \int_{0}^{\infty} e^{-2 \omega s}\|P(-s) x\|^{2} \quad \text { and } \\
& \int_{0}^{\infty} e^{-2 \omega s}\left\|P(s)^{*} x\right\|^{2} d s \leq M_{2}^{2} \int_{0}^{\infty} e^{-2 \omega s}\left\|P(-s)^{*} x\right\|^{2} .
\end{aligned}
$$

Pr oof. From Plancherel's theorem in the Hilbert space $L^{2}(\mathbb{R}, \mathcal{H})$ we see

$$
\begin{aligned}
\int_{0}^{\infty} e^{-2 \omega s}\|P(s) x\|^{2} d s & =\frac{1}{2 \pi} \int_{-\infty}^{\infty}\left\|((\omega+i \xi) I-i A)^{-1} x\right\|^{2} d \xi \\
& \leq M_{2}^{2} \frac{1}{2 \pi} \int_{-\infty}^{\infty}\left\|((\omega-i \xi) I+i A)^{-1} x\right\|^{2} d \xi \\
& =M_{2}^{2} \frac{1}{2 \pi} \int_{-\infty}^{\infty}\left\|((\omega+i \xi) I+i A)^{-1} x\right\|^{2} d \xi \\
& =M_{2}^{2} \int_{0}^{\infty} e^{-2 \omega s}\|P(-s) x\|^{2} d s .
\end{aligned}
$$

For the second equality we apply the previous argument to the dual semigroup.

The following theorem is the analogue (for the group $\{P(t): t \in \mathbb{R}\}$ ) of Theorem 2.3 where we considered the group $\left\{T^{n}: n \in \mathbb{Z}\right\}$.

3.3. TheOrem. Let $A$ be a linear operator with domain and range in a Hilbert space $\mathcal{H}$. The following assertions are equivalent:

(i) The operator $A$ is similar to a self-adjoint operator;

(ii) The operator $i A$ generates a bounded strongly continuous group;

(iii) The operator - iA generates a bounded strongly continuous semigroup, the inverses $(\lambda I-i A)^{-1}$ exist for $\Re \lambda>0$ and

$$
\sup \left\{\Re \lambda\left\|(\lambda I-i A)^{-1}\right\|: \Re \lambda>0\right\}<\infty ;
$$

(iv) The operator -iA generates a strongly continuous semigroup $\{P(t): t \geq 0\}$ with the property that it itself and also its dual semigroup is square bounded in average, and moreover the inverses $(\lambda I-i A)^{-1}, \Re \lambda>0$, exist and

$$
\sup \left\{\Re \lambda\left\|(\lambda I-i A)^{-1}\right\|: \Re \lambda>0\right\}<\infty ;
$$

(v) The operator $-i A$ generates a strongly continuous group $\{P(t): t \in \mathbb{R}\}$, with the property that the semigroup $\left\{P(t)^{*}: t \geq 0\right\}$ as well as the semigroup $\{P(-t): t \geq 0\}$ is square bounded in average;

(vi) The operator $-i A$ is closed, $(\lambda I-i A)^{-1}$ exists for $\Re \lambda \neq 0$ and for each $x$ and $y$ 
in $\mathcal{H}$ the expression

$$
\sup \left\{\omega \int_{-\infty}^{\infty}\left|\left\langle\left(\omega^{2} I+(\xi I-A)^{2}\right)^{-1} x, y\right\rangle\right| d \xi: \omega>0\right\}
$$

is finite and $\lim _{\lambda \rightarrow \infty} \lambda\left\langle(\lambda I+i A)^{-1} x, y\right\rangle=\langle x, y\rangle$.

Pr o of. The equivalence of (i) and (ii) follows from Stone's theorem (see Yosida, l.c.) together with Sz.-Nagy [23]. The implication (ii) $\Rightarrow$ (iii) follows from the representation

$$
(\lambda I-i A)^{-1} x=\int_{0}^{\infty} e^{-\lambda s} P(-s) x d s
$$

where $-i A$ generates the group $\{P(t): t \in \mathbb{R}\}$. Assertion (iii) easily follows from this representation. The implication (iii) $\Rightarrow$ (iv) is not difficult either. The implication (iv) $\Rightarrow(v)$ is a consequence of Proposition 3.2. and of Lemma 1.1. The implication (v) $\Rightarrow(v i)$ may be proved in exactly the same way as we proved Proposition 3.1. The implication (vi) $\Rightarrow$ (ii) remains to be shown. Therefore we define the family of operators $\{P(t): t \in \mathbb{R}\}$ via the equality

$$
\langle P(-t) x, y\rangle=\frac{1}{2 \pi} \int_{-\infty}^{\infty} e^{\omega|t|+i \xi t} \cdot 2 \omega\left\langle\left(\omega^{2} I+(\xi I-A)^{2}\right)^{-1} x, y\right\rangle d \xi .
$$

The expression in (3.6) does not depend on $\omega$, as long as it is strictly positive. So the operators $P(t), t>0$, are well-defined. From a Banach-Steinhaus argument it follows that every operator $P(t)$ is bounded. Moreover we have (for $t>0$ ):

$$
\begin{array}{ll}
\int_{0}^{\infty} e^{-\lambda t}\langle P(-t) x, y\rangle d t & =\left\langle(\lambda I-i A)^{-1} x, y\right\rangle, \quad \Re \lambda>0 \\
\int_{0}^{\infty} e^{-\lambda t}\langle P(t) x, y\rangle d t=\left\langle(\lambda I+i A)^{-1} x, y\right\rangle, & \Re \lambda>0 .
\end{array}
$$

Since the families $\left\{(\lambda I-i A)^{-1}: \Re \lambda>0\right\}$ and $\left\{(\lambda I+i A)^{-1}: \Re \lambda>0\right\}$ are resolvent families it will follow that the family $\{P(s): s \in \mathbb{R}\}$ is a group of operators. Since, finally, $\lim _{\lambda \rightarrow \infty} \lambda\left\langle(\lambda I+i A)^{-1} x, y\right\rangle=\langle x, y\rangle$ it follows that the operator $A$ is densely defined. Since the operators $P(s), s \in \mathbb{R}$, are bounded, the operator $A$ is closed. Hence it is a generator indeed. The inequalities in (3.7) and (3.8) can be established with the aid of Lemma 1.2. Choose the complex measure $\mu_{x, y}$ on $\mathbb{R}$ in such a way that

$$
\begin{aligned}
\frac{1}{2 \pi}\langle((\omega+i \xi) I- & \left.i A)^{-1} x, y\right\rangle+\frac{1}{2 \pi}\left\langle((\omega-i \xi) I+i A)^{-1} x, y\right\rangle \\
& =\frac{\omega}{\pi}\left\langle\left(\omega^{2} I+(\xi I-A)^{2}\right)^{-1} x, y\right\rangle \\
& =\frac{\omega}{\pi} \int_{-\infty}^{\infty} \frac{1}{\omega^{2}+(\xi-\eta)^{2}} d \mu_{x, y}(\eta) \\
& =\frac{1}{2 \pi} \int_{-\infty}^{\infty} \frac{1}{\omega+i \xi-i \eta} d \mu_{x, y}(\eta)+\frac{1}{2 \pi} \int_{-\infty}^{\infty} \frac{1}{\omega-i \xi+i \eta} d \mu_{x, y}(\eta) .
\end{aligned}
$$

Such a choice of the measure $\mu_{x, y}$ is possible by Lemma 1.2(b). By Lemma 2.1(c) we get, 
for $\Re \lambda>0$,

$$
\begin{aligned}
& \left\langle(\lambda I-i A)^{-1} x, y\right\rangle=\int_{-\infty}^{\infty} \frac{1}{\lambda-i \eta} d \mu_{x, y}(\eta), \quad \text { and } \\
& \left\langle(\lambda I+i A)^{-1} x, y\right\rangle=\int_{-\infty}^{\infty} \frac{1}{\lambda+i \eta} d \mu_{x, y}(\eta) .
\end{aligned}
$$

So from (3.6), (3.9), (3.10) and (3.11) together with Cauchy's theorem we obtain

$$
\begin{aligned}
\int_{0}^{\infty} e^{-\lambda t}\langle P(-t) x, y\rangle d t & =\frac{1}{2 \pi i} \int_{\Gamma_{\omega}} \frac{1}{\lambda-z}\left[\left\langle(z I-i A)^{-1} x, y\right\rangle+\left\langle((2 \omega-z) I+i A)^{-1} x, y\right\rangle\right] d z \\
& =\frac{1}{2 \pi i} \int_{\Gamma_{\omega}} \frac{1}{\lambda-z}\left[\int_{-\infty}^{\infty} \frac{1}{z-i \eta} d \mu_{x, y}+\int_{-\infty}^{\infty} \frac{1}{2 \omega-z+i \eta} d \mu_{x, y}\right] d z \\
& =\int_{-\infty}^{\infty} \frac{1}{\lambda-i \eta} d \mu_{x, y}=\left\langle(\lambda I-i A)^{-1} x, y\right\rangle .
\end{aligned}
$$

A similar reasoning will show the equality in (3.8).

Re mark. It is perhaps useful to observe that in the proof of the implication (vi) $\Rightarrow$ (ii) in Theorem 3.3 the Hilbert space $\mathcal{H}$ played only a secondary role. The argument can easily be adapted for a general Banach space instead. In fact the same remark applies to the corresponding implication in Theorem 2.3.

The following example as well as the one of Section 2 is taken from [27].

Example. As in the example at the end of $\S 2$ we fix $0<2 \gamma<1$ and we put $\varphi(x)=(1+|x|)^{\gamma}, x \in \mathbb{R}$. Define for each $s \in \mathbb{R}$ the operator $P(s): L^{2}(\mathbb{R}) \rightarrow L^{2}(\mathbb{R})$ by

$$
P(s) f(x)=\frac{\varphi(x+s)}{\varphi(x)} f(x+s), \quad x \in \mathbb{R}, f \in L^{2}(\mathbb{R}) .
$$

Then the family $\{P(s): s \in \mathbb{R}\}$ is a strongly continuous group for which $\|P(s)\|=\varphi(s)$ and for which the expression

$$
\sup \left\{\frac{1}{2 t} \int_{-t}^{t}\|P(s) f\|^{2} d s: t>0\right\}
$$

is finite. Define the operator $A$ as follows. Its domain $D(A)$ is given by $D(A)=\{f \in$ $\left.L^{2}(\mathbb{R}): f^{\prime} \in L^{2}(\mathbb{R})\right\}$ and $A f, f \in D(A)$, is given by

$$
A f(x)=i f^{\prime}(x)+\frac{i \gamma x}{|x|} \frac{1}{1+|x|} f(x), \quad x \in \mathbb{R} .
$$

Then $-i A$ generates the group $\{P(s): s \in \mathbb{R}\}$. Since $\sup \{\|P(s)\|: s \in \mathbb{R}\}=\infty$, the operator $A$ cannot be similar to a self-adjoint one.

4. Bounded families of operators on a Banach space. In this section we will especially be interested in boundedness properties of operators $T$, defined on a Banach space $X$, with their spectrum in the closed unit disc and for which there exist a finite constant $M_{3}$ with the property that

$$
(1-|\lambda|)\left\|(I-\lambda T)^{-1}\right\| \leq M_{3}, \quad|\lambda|<1 .
$$


In addition we will discuss some corresponding boundedness properties for strongly continuous semigroups. In fact let $A$ be the generator of a strongly continuous semigroup $\{P(t): t \geq 0\}$ of linear operators on $X$ with the property that

$$
\Re \lambda\left\|(\lambda I-A)^{-1}\right\| \leq M_{4}, \quad \Re \lambda>0 .
$$

A problem could be to describe the growth of $\|P(t)\|$ as $t$ goes to infinity. In case of a single operator we will show that $\left\|T^{n}\right\|=\mathcal{O}(n), n \rightarrow \infty$, and that this bound is optimal. All this provided we have a bound like in (4.1). Essentially the same phenomenon occurs for semigroups. We begin with the discrete case. The result in Proposition 4.1 coincides with Theorem 6.1 in Strikwerda [22].

4.1. Proposition. Let $T$ be a continuous linear operator in a Banach space that obeys (4.1). Then the following inequalities are valid:

$$
\begin{aligned}
(1-|\lambda|)\left\|(I-\lambda T)^{-1}\right\| & \leq 2 \sup _{n \in \mathbb{N}} \frac{\left\|\sum_{k=0}^{n}(n+1-k)(\mu T)^{k}\right\|}{(n+1)(n+2)} \\
& \leq e \sup _{\lambda \in U}(1-|\lambda|)\left\|(I-\lambda T)^{-1}\right\| .
\end{aligned}
$$

Here $\mu=\lambda /|\lambda|$ and $U$ is the open unit disc in $\mathbb{C}$. In fact, for functions harmonic on the unit disc $U$, a much more general result was obtained by Bennett, Stegenga and Timoney in [3, Theorem 1.4]. For related results see Anderson, Clunie and Pommerenke [1] and Anderson and Shields [2].

Proof of Proposition 4.1. Fix $z \in U$ and let $\mu$ be a complex number of absolute value 1 . Then the following identity is true:

$$
(I-\mu z T)^{-1}=(1-z)^{2} \sum_{n=0}^{\infty} \sum_{k=0}^{n}(n+1-k)(\mu T)^{k} z^{n} .
$$

So with $z=|\lambda|$ and $\mu$ as above we infer the first inequality in (4.1). In order to obtain the second inequality we use Cauchy's theorem to get the following identity for $r=n /(n+1)$ and $\mu \in \mathbb{C},|\mu|=1$ :

$$
\sum_{k=0}^{n}(n+1-k)(\mu T)^{k}=\frac{1}{2 \pi} \int_{-\pi}^{\pi} \frac{1}{r^{n} e^{i n \vartheta}}\left(I-\mu r e^{i \vartheta} T\right)^{-1} \frac{d \vartheta}{\left(1-r e^{i \vartheta}\right)^{2}}
$$

and so

$$
\begin{aligned}
\left\|\sum_{k=0}^{n}(n+1-k)(\mu T)^{k}\right\| & =M_{3} \frac{1}{r^{n}} \frac{1}{1-r} \frac{1}{2 \pi} \int_{-\pi}^{\pi} \frac{1}{1-2 r \cos \vartheta+r^{2}} d \vartheta \\
& =M_{3} \frac{1}{r^{n}} \frac{1}{1-r} \frac{1}{1-r^{2}} \leq e M_{3}(n+1)(n+2),
\end{aligned}
$$

where $M_{3}=\sup \left\{(1-|\lambda|)\left\|(I-\lambda T)^{-1}\right\|: \lambda \in U\right\}$. This shows the inequalities in (4.1).

4.2. Theorem. Let $T$ be a bounded linear operator on a Banach space $X$. Suppose that the spectrum of $T$ is contained in the closed unit disc $U$ of $\mathbb{C}$. Put $M(r)=\sup \{(1-$ $\left.|\lambda|)\left\|(I-\lambda T)^{-1}\right\|:|\lambda| \leq r\right\}$. The following assertions hold true:

(i) $\left\|T^{n}\right\| \leq(n+1)(1+1 / n)^{n} M(n /(n+1)), n \in \mathbb{N}$;

(ii) If $M(1 / 2) \leq 1$, then $\left\|T^{n}\right\| \leq 2 \sqrt{\pi} \Gamma(n+1) / \Gamma(n+1 / 2), n \in \mathbb{N}$; 
(iii) If $M(r) \leq 1,0<r<1$, then $\left\|T^{n}\right\| \leq n ! e^{n} n^{-n}, n \in \mathbb{N}$;

(iv) If $M_{3}=\sup \{M(r): 0<r<1\}$ is finite, then $\left\|T^{n}\right\| \leq e M_{3}(n+1), n \in \mathbb{N}$.

Most of the results in Theorem 4.2 are well-known: see e.g. Shields [19] and the references given there $\left(^{1}\right)$. Nevertheless we indicate the main ingredients of a proof.

Proof of Theorem 4.2. (i) By Cauchy's integral formula we have

$$
T^{n}=\frac{1}{2 \pi i} \int_{\{|\lambda|=\rho\}}(I-\lambda T)^{-1} \lambda^{-n-1} d \lambda, \quad 0<\rho<1 .
$$

With $\rho=n(n+1)^{-1}$ assertion (i) follows.

(ii) Notice that

$$
(I-\lambda T)^{-n-1}=\sum_{k=0}^{\infty}\left(\begin{array}{c}
k+n \\
k
\end{array}\right) \lambda^{k} T^{k}, \quad|\lambda|<1 .
$$

From Cauchy's integral formula it follows that

Consequently,

$$
\left(\begin{array}{c}
2 n \\
n
\end{array}\right) T^{n}=\frac{1}{2 \pi i} \int_{\{|\lambda|=1 / 2\}}(I-\lambda T)^{-n-1} \lambda^{-n-1} d \lambda .
$$

$$
4^{n} \frac{\Gamma\left(n+\frac{1}{2}\right)}{\Gamma(n+1)} \frac{\left\|T^{n}\right\|}{\sqrt{\pi}} \leq 4^{n} M(1 / 2)^{n+1} .
$$

So (ii) follows.

(iii) If $M(r) \leq 1,0<r<1$, then $\left\|(I-\lambda T)^{-n}\right\| \leq(1-|\lambda|)^{-n}, \lambda \in U$, and so

$$
\left\|\left(I-\frac{\lambda}{n} T\right)^{-n}\right\| \leq\left(1-\frac{|\lambda|}{n}\right)^{-n}, \quad|\lambda|<n .
$$

Consequently, $\|\exp (\lambda T)\| \leq \exp (|\lambda|), \lambda \in \mathbb{C}$. Applying Cauchy's formula once more we get

$$
\frac{T^{n}}{n !}=\frac{1}{2 \pi i} \int_{\{|\lambda|=n\}} \frac{\exp (\lambda T)}{\lambda^{n+1}} d \lambda .
$$

Hence (iii) follows.

(iv) This assertion follows trivially from (i).

Remark 1. In [19] Shields gives an example of an operator $T$ in a Banach space for $\left\|T^{n}\right\|=n+1$ and for which (4.1) is satisfied. This kind of operators are in Shields' terminology Möbius bounded. In fact for the Banach space $X$ we may take $X=H^{\infty}(U) \cap$ $H^{1}(U)$ supplied with the norm given by $\|f\|=\|f\|_{\infty}+\|f\|_{1}, f \in X$. The operator $T$ is given by $[T f](z)=z f(z), f \in X$. Another example of this kind is obtained if for $X$ we take the space $X_{\text {Bloch }}$ of derivatives of Bloch functions, i.e. a function $f$ belongs to $X_{\text {Bloch }}$ if it is analytic on the open unit disc $U$ and if $\|f\|_{\text {Bloch }}=\sup \{(1-|z|)|f(z)|: z \in U\}<\infty$, $f \in X$. The operator $T$ is now given by $[T f](z)=(f(z)-f(0)) / z, z \in U, f \in X_{\text {Bloch }}$. Then it is not so difficult to show that

$$
(1-|\lambda|)\left\|(I-\lambda T)^{-1} f\right\|_{\text {Bloch }} \leq 4\|f\|_{\text {Bloch }}, \quad f \in X_{\text {Bloch }},
$$

\footnotetext{
$\left({ }^{1}\right)$ Editorial note: See also the paper by O. Nevanlinna in this volume.
} 
and

$$
(n+1)\left(1+\frac{1}{n}\right)^{n} \leq\left\|T^{n}\right\| \leq 4(n+1)\left(1+\frac{1}{n}\right)^{n}, \quad n=1,2, \ldots
$$

Moreover, if $f(z)=\sum_{k=0}^{\infty} a_{k} z^{k}$ belongs to $X$, then $a_{n}=T^{n} f(0)$. In addition there exists a constant $C$ such that $\left|\sum_{j=0}^{n} a_{j} z^{j}\right| \leq C(n+1) \log (n+1)\|f\|_{\text {Bloch }}$ for all $f \in X_{\text {Bloch }}$, for all $n \in \mathbb{N}$, and for all $z \in U$. Moreover this estimate is best possible: see Bennett et al. [3, Theorem 1.12. p. 523]. For more details on Bloch functions see e.g. Anderson, Clunie and Pommerenke [1] and Anderson and Shields [2].

Remark 2. In [5] Bonsall and Duncan describe an example, due to Crabb, of a Banach algebra containing an element $u$ for which $\|\exp (\lambda u)\| \leq \exp (|\lambda|), \lambda \in \mathbb{C}$, and for which $\left\|u^{n}\right\|=n ! e^{n} n^{-n}, n \in \mathbb{N}$. In fact our assumption (4.1) with $M_{3}=1$ is equivalent to saying that the numerical radius of $T$ is less than or equal to 1 ; see Bonsall and Duncan [4, Theorem 10, 11, pp. 54-55].

Remark 3 . If $X$ is a Hilbert space, if $S=T^{-1}$ exists and if $\|S\| \leq 1$, then

$$
\sup \left\{\left\|T^{n}\right\|: n \in \mathbb{N}\right\}
$$

is finite if and only if $T$ is Möbius bounded, i.e. if and only if

$$
\sup \left\{(1-|\lambda|)\left\|(I-\lambda T)^{-1}\right\|: \lambda \in U\right\}
$$

is finite: see Corollary 1.4, p. 335 in Sz.-Nagy and Foiaş [23]. In fact in Theorem 2.3 we proved a stronger statement.

Remark 4. In [12, Theorem 3.3. p. 46] Hayman exhibits a class of holomorphic functions on the unit disc for which the expression $\sup \{(1-|z|)|F(z)|: z \in U\}$ is finite if and only if its Taylor coefficients constitute a bounded sequence. In general this is not true. For example the function $z \mapsto \sum_{n=0}^{\infty} 2^{n} z^{2^{n}}, z \in U$, belongs to the space $X_{\text {Bloch }}$ defined in Remark 1. In fact a function $f(z)=\sum_{k=0}^{\infty} a_{k} z^{k}$ belongs to $X_{\text {Bloch }}$ if and only if the expression

$$
\sup \left\{\frac{1}{(n+1)(n+2)}\left|\sum_{k=0}^{n}(n-k) \mu^{k} a_{k}\right|:|\mu| \leq 1, n \in \mathbb{N}\right\}
$$

is finite. A proof can be patterned after the proof of Proposition 4.1. In [3, Theorem 1.4] this kind of result was generalized to a considerable extent.

Next we consider a strongly continuous semigroup $\{P(t): t \geq 0\}$ with generator $A$ and resolvent family $\{R(\lambda): \Re \lambda>0\}$. We assume that

$$
R(\lambda) x=\int_{0}^{\infty} e^{-\lambda t} P(t) x d t=(\lambda I-A)^{-1} x
$$

exists for each $x \in X$ and for every $\lambda \in \mathbb{C}$ with $\Re \lambda>0$.

4.3. TheOREM. Let $\{P(t): t \geq 0\}$ be a strongly continuous semigroup with generator $A$ and resolvent $\{R(\lambda): \Re \lambda>0\}$. Put $M_{4}=\sup \{\Re \lambda\|R(\lambda)\|: \Re \lambda>0\}$. Then the equality

$$
\|P(t) x\| \leq 2 M_{4} e(1+t)\left\|(I-A)^{2} x\right\|
$$

holds for each $t>0$ and for each $x \in D\left(A^{2}\right)$. 
Pr oof. Let $\lambda \in \mathbb{C}$ have a strictly positive real part. From Theorem II.6.1, p. 349 in Hille and Phillips [13] together with the resolvent identity we infer

$$
P(t) R(\lambda)^{2} x=\frac{1}{2 \pi} \int_{-\infty}^{\infty} \frac{e^{\omega t+i \xi t}}{(\lambda-\omega-i \xi)^{2}} R(\omega+i \xi) x d \xi, \quad 0<\omega<\Re \lambda
$$

Therefore

$$
(\Re \lambda)^{2}\left\|P(t) R(\lambda)^{2} x\right\| \leq \frac{1}{2} M_{4} \frac{e^{\omega t} t^{2}(\Re \lambda)^{2}}{\omega t(t \Re \lambda-\omega t)}\|x\|
$$

and hence, upon optimizing $\omega$,

$$
(\Re \lambda)^{2}\left\|P(t) R(\lambda)^{2} x\right\| \leq M_{4} e^{1+s-\sqrt{1+s^{2}}}\left(1+\sqrt{1+s^{2}}\right)\|x\|,
$$

where $2 s=t \Re \lambda$. With $\lambda=1$ we get the result in Theorem 4.3.

In the following example we describe, without proof, a continuous analogue of the space $X_{\text {Bloch }}$ of Remark 1 . For the proofs some standard facts on Laplace transforms of measures are used: see Widder [29, 30].

ExAMPLE. Let $X$ be the space of all holomorphic functions $f$ on the open right half plane for which

$$
\|f\|:=\sup \{\Re z|f(z)|: \Re z>0\}
$$

is finite. Then $X$, equipped with this norm, is a Banach space. On $X$ we define a resolvent family $\{R(\lambda): \Re \lambda>0\}$ as follows:

$$
R(\lambda) f(z)= \begin{cases}\frac{f(z)-f(\lambda)}{\lambda-z} & \text { for } z \neq \lambda \\ -f^{\prime}(\lambda) & \text { for } z=\lambda .\end{cases}
$$

Each operator $R(\lambda), \Re \lambda>0$, maps $X$ into itself. On the space $R(\lambda)^{2} X$ we define the semigroup $\{P(t): t \geq 0\}$ as follows:

$$
\begin{aligned}
P(t) R(\lambda)^{2} f(z) & =\frac{1}{2 \pi i} \int_{\omega-i \infty}^{\omega+i \infty} \frac{e^{w t}}{(\lambda-w)^{2}} \frac{f(z)-f(w)}{w-z} d w \\
& =\frac{1}{2 \pi i} \int_{\omega-i \infty}^{\omega+i \infty} e^{w t} R(w) R(\lambda)^{2} f(z) d w
\end{aligned}
$$

for $0<\omega<\Re \lambda$ and $\Re z>0$. For $\alpha>0$ we put

$$
\mathcal{X}_{\alpha}=\left\{f \in R(\lambda)^{2} X: \sup _{t>0} e^{-\alpha t}\|P(t) f\|<\infty\right\} .
$$

The space $X_{\alpha}$ will be the completion of $\mathcal{X}_{\alpha}$ with respect to the norm $\|\cdot\|_{\alpha}$, defined by

$$
\|f\|_{\alpha}=\sup _{t>0} e^{-\alpha t}\|P(t) f\|, \quad f \in \mathcal{X}_{\alpha} .
$$

Since $\|f\|_{\alpha} \geq\|f\|$, for $f \in \mathcal{X}_{\alpha}$, it follows that $X_{\alpha}$ is a subspace of $X$. Then the semigroup $\{P(t): t \geq 0\}$ defined on $\mathcal{X}_{\alpha}$ can be extended to a strongly continuous semigroup on $X_{\alpha}$. We claim that the latter semigroup, again denoted by $\{P(t): t \geq 0\}$, has a number of interesting properties, which may serve as an example or counter-example. Among others the semigroup $\{P(t): t \geq 0\}$ possesses the following properties:

(1) If $f$ belongs to $X$, then $\Re \lambda\|R(\lambda) f\| \leq 2\|f\|, \Re \lambda>0$.

(2) If $f$ belongs to $X_{\alpha}$, then $\Re \lambda\|R(\lambda) f\|_{\alpha} \leq 2\|f\|_{\alpha}, \Re \lambda>0$. 
(3) For $f \in X_{\alpha}$ the inequality $\|P(s) f\|_{\alpha} \leq e^{\alpha s}\|f\|_{\alpha}$ is valid.

(4) Let $f$ belong to $X$ and put

$$
\varphi(t)=\frac{1}{2 \pi i} \int_{\omega-i \infty}^{\omega+i \infty} \frac{e^{z t}}{z^{2}} f(z) d z, \quad t \geq 0, \omega>0 .
$$

Then

$$
f(z)=z^{2} \int_{0}^{\infty} e^{-z s} \varphi(s) d s, \quad \Re z>0 .
$$

If, in addition, $f$ belongs to $X_{\alpha}, \varphi$ is differentiable, and $\varphi^{\prime}(0)=0$, then

$$
P(t) f(z)=z^{2} \int_{0}^{\infty} e^{-z s} Q(t) \varphi(s) d s, \quad \Re z>0, t \geq 0 .
$$

Here $Q(t) \varphi(s)=\varphi(s+t)-\varphi(t)-s \varphi^{\prime}(t), s, t \geq 0$.

(5) Let $f$ in $X_{\alpha}$ be of the form $f(z)=\int_{0}^{\infty} e^{-z s} \varphi(s) d s$, where $\varphi$ is a continuous function. Then

$$
P(t) f(z)=\int_{0}^{\infty} e^{-z s} f(s+t) d s, \quad \Re z>0, t \geq 0,
$$

and

$$
\|P(t) f\| \geq|\varphi(t)|, \quad t \geq 0 .
$$

(6) Let $A$ be the generator of the semigroup $\{P(t): t \geq 0\}$ defined on $X_{\alpha}$ and suppose that $f$ belongs to $D(A)$. Then $\lim _{\lambda \rightarrow \infty} \lambda G(\lambda)$ exists and the function $g$ defined by $g(z)=z f(z)-\lim _{\lambda \rightarrow \infty} \lambda f(\lambda), \Re z>0$, belongs to $X_{\alpha}$. Moreover $A f=g$.

(7) Let $\varphi$ be a continuous function on $[0, \infty)$ for which its Laplace transform $f$, defined by $f(z)=\int_{0}^{\infty} e^{-z s} \varphi(s) d s$, belongs to $X$. Put $\psi(s)=\int_{0}^{\infty} e^{-\sigma} \sigma f(\sigma+s) d \sigma, \sigma \geq 0$, and $g(z)=\int_{0}^{\infty} e^{-z s} \psi(s) d s, \Re z>0$. Then $g$ belongs to $R(\lambda)^{2} X$ and in fact $g=R(1)^{2} f$ and $\varphi=\psi-2 \psi^{\prime}+\psi^{\prime \prime}$.

(8) Put $u(s)=\max (1-|s|, 0), s \in \mathbb{R}$. Write $\varphi_{t}(s)=t u(2(s-t-1 / 2)), s \geq 0$, and $\psi_{t}(s)=\int_{0}^{\infty} \sigma e^{-\sigma} \varphi_{t}(\sigma+s) d \sigma, s \geq 0$. Suppose that $f_{t}$ and $g_{t}$ denote the Laplace transforms of $\varphi_{t}$ and $\psi_{t}$ respectively. Then $f_{t}$ belongs to $R(\lambda)^{2} X, \Re \lambda>0$, and $R(1)^{2} f_{t}=g_{t}$. Moreover the following inequalities hold:

$$
\frac{1}{4}\left(1-\frac{1}{\sqrt{e}}\right)\left(8-\frac{11}{\sqrt{e}}\right) t \leq\left\|P(t) g_{t}\right\|_{\alpha} \leq 4 e(t+1)\left\|f_{t}\right\|_{\alpha} \leq 4(t+1) \max \left(\alpha^{-1}, 1\right) .
$$

\section{References}

[1] J. M. Anderson, J. G. Clunie and Ch. Pommerenke, On Bloch functions and normal families, J. Reine Angew. Math. 270 (1974), 12-37.

[2] J. M. Anderson and A. L. Shields, Coefficient multipliers of Bloch functions, Trans. Amer. Math. Soc. 224 (1976), 255-265.

[3] G. Bennett, D. A. Stegenga and R. M. Timoney, Coefficients of Bloch functions and Lipschitz functions, Illinois J. Math. 25 (1981), 520-531.

[4] F. F. Bonsall and J. Duncan, Complete Normed Algebras, Springer, Berlin, 1973. 
[5] F. F. Bonsall and J. Duncan, Numerical Ranges II, London Math. Soc. Lecture Note Ser. 10, Cambridge University Press, 1973.

[6] P. Duren, Theory of $H^{p}$-spaces, Academic Press, New York, 1970.

[7] R. E. Edwards and G. I. Gaudry, Littlewood-Paley and Multiplier Theory, Springer, Berlin, 1977.

[8] H. Hewitt and K. A. Ross, Abstract Harmonic Analysis I, Springer, Berlin, 1974.

[9] H. G. Garnir, K. R. Unni and J. H. Williams, Functional Analysis and its Applications, Lecture Notes in Math. 399, Springer, Berlin, 1974.

[10] I. C. Gohberg and M. G. Krein, On a description of contraction operators similar to unitary ones, Funktsional. Anal. i Prilozhen. 1 (1) (1976), 38-60 (in Russian).

[11] P. R. Halmos, A Hilbert Space Problem Book, Van Nostrand, Princeton, N.J., 1976.

[12] W. K. Hayman, Multivalent Functions, Cambridge University Press, Cambridge, 1958.

[13] E. Hille and R. S. Phillips, Functional Analysis and Semi-groups, Amer. Math. Soc., Providence R.I., 1957.

[14] T. Kato, Perturbation Theory for Linear Operators, Springer, Berlin, 1976.

[15] M. G. Krein, Analytic problems in the theory of linear operators in Hilbert space (abstract), Amer. Math. Soc. Transl. 70 (1968), 68-72.

[16] - Analytic problems in the theory of linear operators in Hilbert space, in: Proc. Internat. Congress Math. Moscow 1966, Mir, Moscow, 1968, 189-216 (in Russian).

[17] M. Mbekhta and J. Zemánek, Sur le théorème ergodique uniforme et le spectre, C. R. Acad. Sci. Paris Sér. I Math. 317 (1993), 1155-1158.

[18] W. Rudin, Real and Complex Analysis, 2nd ed., McGraw-Hill, New York, 1974.

[19] A. L. Shields, On Möbius bounded operators, Acta Sci. Math. (Szeged) 40 (1978), $371-374$.

[20] J. G. Stampfli, A local spectral theory for operators, III: Resolvents, spectral sets and similarity, Trans. Amer. Math. Soc. 168 (1972), 133-151.

[21] E. M. Stein, Singular Integral Operators and Differentiability Properties of Functions, Princeton Math. Ser. 30, Princeton University Press, 1970.

[22] J. C. Strikwerda, Finite Differences and Partial Differential Equations, The Wadsworth and Brooks/Cole Math. Series vol. 1989 (1), Wadsworth \& Brooks, Pacific Grove, Calif., 1989.

[23] B. Sz.-Nagy, On uniformly bounded linear transformations in Hilbert space, Acta Sci. Math. (Szeged) 11 (1947), 152-157.

[24] B. Sz.-Nagy and C. Foiaş, Sur les contractions de l'espace de Hilbert X; contractions similaires à des transformations unitaires, ibid. 26 (1965), 79-91.

[25] —, 一, Analyse Harmonique des Opérateurs de l'Espace de Hilbert, Akadémiai Kiadó, Budapest, 1967.

[26] J. A. van Casteren, A problem of Sz.-Nagy, Acta Sci. Math. (Szeged) 42 (1980), 189-194.

[27] - Operators similar to unitary or self-adjoint ones, Pacific J. Math. 104 (1983), 241-255.

[28] - Generators of Strongly Continuous Semigroups, Pitman, London, 1985.

[29] D. V. Widder, The Laplace Transform, Princeton University Press, Princeton, 1972.

[30] - An Introduction to Transform Theory, Academic Press, New York, 1971.

[31] K. Yosida, Functional Analysis, 3rd ed., Springer, Berlin, 1971.

[32] J. Zemánek, On the Gelfand-Hille theorems, in: Functional Analysis and Operator Theory, Banach Center Publ. 30, Inst. Math., Polish Acad. Sci., 1994, 369-385. 